\title{
Dynamics Study of Constant DI and Constant TR Control for Cardiac Alternans Based on a Two Dimensional Cellular Automata Model
}

\section{Min Xiong}

University of Tennessee Knoxville College of Engineering: The University of Tennessee Knoxville Tickle College of Engineering

\section{Kai Sun}

University of Tennessee Knoxville College of Engineering: The University of Tennessee Knoxville Tickle College of Engineering

\section{Xiaowen Su}

University of Tennessee Knoxville College of Engineering: The University of Tennessee Knoxville Tickle College of Engineering

\section{Elena G. Tolkacheva}

University of Minnesota Twin Cities Campus: University of Minnesota Twin Cities

Xiaopeng Zhao ( $\sim$ xzhao9@utk.edu )

University of Tennessee Knoxville College of Engineering: The University of Tennessee Knoxville Tickle College of Engineering https://orcid.org/0000-0003-1207-5379

\section{Research Article}

Keywords: Cardiac arrhythmias, alternans, conduction block, constant DI control, ECG, constant TR control, cellular automata model

Posted Date: December 8th, 2021

DOI: https://doi.org/10.21203/rs.3.rs-1130154/v1

License: (1) (1) This work is licensed under a Creative Commons Attribution 4.0 International License.

Read Full License 


\title{
Dynamics study of constant DI and constant TR control for cardiac alternans based on a two dimensional cellular automata model
}

\author{
Min Xiong ${ }^{1}$, Kai Sun ${ }^{1}$, Xiaowen $\mathrm{Su}^{1,2}$, Elena G. Tolkacheva ${ }^{3}$, Xiaopeng Zhao ${ }^{2}$ \\ ${ }^{1}$ Department of Electrical Engineering and Computer Science, University of Tennessee, \\ Knoxville, $T N$ \\ ${ }^{2}$ Department of Mechanical, Aerospace, and Biomedical Engineering, University of Tennessee, \\ Knoxville, $T N$ \\ ${ }^{3}$ Department of Biomedical Engineering, University of Minnesota, Minneapolis, MN \\ mxiong3@vols.utk.edu,kaisun@utk.edu,xsu2@vols.utk.edu, talkacal@umn.edu,xzhao9@utk.edu
}

\begin{abstract}
As a precursor for cardiac arrhythmias such as atrial and ventricular fibrillations, which could cause sudden cardiac death (SCD), cardiac alternans is essentially an unstable heart rhythm with alternating long and short action potential durations (APD) of cardiac myocytes that usually occurs under fast pacing conditions. In this paper, the constant TR control method based on global pseudo-electrocardiogram (ECG) is studied and compared with the local constant diastolic interval (DI) control method using a 2-dimensional (2-D) cellular automata model (CAM), aiming at preventing or eliminating cardiac alternans before arrhythmias. The results show that both the constant TR and constant DI control methods are effective in stabling the alternans to a smaller basic cycle length (BCL). Also, the efficacy of the two control approaches depends on the "decrease step" $\Delta$ in the downsweep protocol, and a smaller $\Delta$ could significantly improve their performance. Besides, in general, constant TR control is superior to constant DI control in alternans prevention when a relatively large $\Delta$ is adopted.
\end{abstract}

Keywords: Cardiac arrhythmias; alternans; conduction block; constant DI control; ECG; constant TR control; cellular automata model.

\section{INTRODUCTION}

Cardiac alternans is a disturbed heart rhythm in which the action potential duration (APD) of cells alternates between long and short values, and manifests on electrocardiograms (ECG) as alternantion in amplitude between two consecutive T-waves ${ }^{[1]}$. In experimental and clinical practice, cardiac alternans could be a sensitive indicator of arrhthmias leading to life-threatening sudden cardiac death (SCD), which accounts for the death of around 325,000 Americans per 
year. Thus, understanding and preventing cardiac alternans has been a research topic of great significance $^{[2]}$.

Based on the restitution theory, which has been widely used in cardiac alternans research, the APD of a cell in one cycle is a function of its diastolic interval (DI) in previous cycle, as shown in Eq. (1), where DI is the difference between the basic cycle length (BCL) and APD in the same cycle.

$$
A P D_{n+1}=f\left(D I_{n}\right)
$$

where $f$ is the restitution curve.

Then, as shown in Fig. 1(a), under a constant BCL or so-called periodical pacing protocol, when the $\mathrm{BCL}$ is long (e.g., $\mathrm{BCL}=\mathrm{BCL}_{1}$ ), the cardiac response is normal and $\mathrm{APD}$ is locked as 1:1 rhythm, and at steady state

$$
\begin{gathered}
A P D_{1}=f\left(D I_{1}\right) \\
B C L_{1}=A P D_{1}+D I_{1}
\end{gathered}
$$

Also, as shown in Fig. 1(b) and Fig. 1(c), APD alternates between $\mathrm{APD}_{2}$ and $\mathrm{APD}_{3}$ when $\mathrm{BCL}=\mathrm{BCL}_{2}$ is small and then we have:

$$
\begin{gathered}
A P D_{2}=f\left(D I_{2}\right) \\
A P D_{3}=f\left(D I_{3}\right) \\
B C L_{2}=A P D_{2}+D I_{3}=A P D_{3}+D I_{2}
\end{gathered}
$$

Besides, Eq. (1) indicates that alternans occurs when slope of the restitution curve is smaller than $1^{[3],[4]}$. As shown in Fig. 1(c) and Fig. 1(d), APD alternates when BCL is smaller than BCL ONSET $^{\mathrm{BCL}}$, and stable normal response occurs when $B C L$ is larger than $\mathrm{BCL}_{\mathrm{ONSET}}^{\mathrm{BC}}$, $S O \mathrm{BCL}_{\mathrm{ONSET}}^{\mathrm{BC}}$ is a bifurcation point.

To suppress cardiac alternans, different control methods have been proposed and tested in the past decades using both numerical models and/or experiments on isolated animal hearts. An adaptive proportional feedback control approach was proposed and tested on rabbit hearts in [4]. In [5], an alternating-period feedback control mechanism was tested through numerical simulation on a single cell and a 1-dimensional (1-D) tissue. Besides, the effect of a feedback control method was studied on isolated canine Purkinje fibers in [6]. However, feedback control methods are generally ineffective for tissue with a distance longer than $2 \mathrm{~cm}$ due to complex 
spatiotemporal electrophysiological dynamics, and stimulation from multiple sites are required for large tissues, also, it is difficult to determine optimal control parameters ${ }^{[7]-[9]}$.

Due to the limited success of the feedback control, which utilises a beat-to-beat regulation of the BCL, a constant DI control method that decouples the relation between APD and DI was adopted to better suppress alternans ${ }^{[10]}$. As shown in Fig. 2, constant DI pacing protocol keeps the DI of the cell constant based on measured transmembrane voltage of the $1^{\text {st }}$ cell in a tissue. In this way, the instability caused by change of APD can be eliminated, thus the pacing rhythm could be easier stabilized. Constant DI control was employed and tested in single-cell models ${ }^{[10],[11]}$ and ventricular fibers models ${ }^{[12],[13]}$ based on numerical simulation and/or experiments. However, these studies have validated efficacy of the constant DI control only on 0-D or 1-D tissues, and demonstrated that control may fail on 1-D cables and 2-D tissues with large size due to complex spatiotemporal evolution of cardiac activity ${ }^{[14],[15]}$. In addition, it is challenging to apply constant DI control in experiments due to the difficulty of performing real-time APD and DI measurements ${ }^{[15]}$. So, it is important to develop other control approaches with higher efficacy. As is known, the APD alternans can be observed on ECG as T-wave alternans which is easier to be detected in real-time ${ }^{[16],[17]}$, and the TR interval can be regarded as a global DI. Thus, similar to the concept of constant DI control, a constant TR control method was proposed and employed in recent research ${ }^{[15],[17],[18]}$. As shown in Fig. 3, under constant TR pacing, based on the ECG of the whole tissue, stimulations are added after detection of T peaks to keep TR intrvels on the ECG constant, and therefore the constant TR protocol can be regarded as a "globalized" version of the constant DI protocol. In [15], the antiarrhythmic effects of constant TR control were validated using experiments on ex vivo whole rabbit hearts, yet there are some limitations such as accurate detection of a distinct T-wave and size of the heart. In [17], based on a 1-D numerical model of human ventricular tissue $(<7 \mathrm{~cm})$, the efficacy of constant TR control of alternans was studied and compared with that of constant DI control. The results show that the constant TR control is superior for alternans control in longer fibers when compared to constant DI pacing. Nevertheless, the simulation efficiency was low, and the study has not been performed on a higher dimensional (e.g., 2-D) model with a larger size.

Thus, it is of great significance to efficiently study cardiac alternans control on a high dimensional model with large sizes, and a cellular automata model (CAM) is a good choice to achieve this goal. As a powerful model, which has successful applications in biology, physics, 
and other engineering areas, a CAM uses simple rules to compute complex phenomena with fewer calculations, and the process can be easily visualized to provide an intuitive illustration of system dynamics ${ }^{[19]}$. Although CAMs are less accurate than detailed models and vivo animal hearts experiments, they can represent the main features and key dynamical behaviors of the cardiac tissue. Compared with regular full physiological models, which require extensive computation, CAMs are much more efficient. Also, compared with ex vivo hearts, CAMs are more convenient and economical for tests on tissues of different sizes.

In this paper, we tested the efficacy of both the constant DI and constant TR control on alternans prevention using a 2-D CAM.

\section{METHODS}

\section{Two-Dimensional CAM for Cardiac Simulation}

Both 2-D and 3-D CAMs have been developed to simulate cardiac electrical wave propagation and fibrillation ${ }^{[20]-[26]}$; however, dynamical phenomena related to cardiac alternans have never been studied in these publications. Recently, a 2-D CAM capable of simulating cardiac dynamics including ectopic beat, wave break, spiral wave, conduction block, and alternans was proposed by our group ${ }^{[27]}$. Here, we adopted the same model with better practical parameter settings for the study of cardiac alternans control. Detailed mechanism of the employed 2-D CAM is described below.

\section{Wave Propagation Rule in the CAM}

To simulate dynamics of a 2-D cardiac tissue, an $n \times n$ CAM is constructed by MATLAB, and each small square in the CAM represents an individual cell of the tissue. Then, based on their transmembrane voltages, the cell states can be divided into four categories as listed in Table ${ }^{[20]}$, where the transmembrane voltage of a cell becomes 1 when it is excited or stimulated. A $3 \times 3$ square, which contains 9 cells in the bottom left corner of the CAM, acts as the paced cells, and they excite according to some protocols such as periodic pacing. Besides, as shown in Fig. 4, a single cell (black square) is excited at next time step, if its transmembrane voltage is less than $0.1 \mathrm{~V}$ and the voltages of at least three adjacent cells (gray squares) are bigger than 0.9 
$\mathrm{V}^{[26]}$. Otherwise, its transmembrane voltage just evolves with time according to the voltage waveform function, which will be introduced below.

\section{Transmembrane Voltage Waveform Function}

The transmembrane voltage of a cell will suddenly change to $1.0 \mathrm{~V}$ the moment it is excited, otherwise, its voltage will progress according to the function below:

$$
\begin{aligned}
& V(A P D, t)=\frac{e^{-t / T(A P D)}}{c+e^{-t / T(A P D)}} \\
& T(A P D)=\frac{A P D}{\ln (0.9)-\ln (0.1 \times c)}
\end{aligned}
$$

where $t$ is the time elapsed since last stimulation; $c$ is a constant number which ensures $V(A P D, t=0) \approx 1.0 \mathrm{~V}$ and is set as 0.001 in this model; $A P D$ is determined by a restitution curve which will be given in next section. Thus, when the APD is determined, the parameter $T$ can be calculated by Eq. (5), and then transmembrane voltage $V$ in Eq.(4) will decrease from 1 to nearly 0 with the increase of $t$.

In addition, it can be easily proved or calculated that $V(A P D, t=A P D)=0.1 \mathrm{~V}$, which is exactly the refractory threshold. This also helps to define that the APD in this CAM is the time duration for the voltage to decrease from $1 \mathrm{~V}$ to refractory threshold $0.1 \mathrm{~V}$.

For example, when APD=300 ms, the transmembrane voltage waveform given by Eq. (4)-(5) is shown in Fig. 5.

\section{Restitution Curve}

To provide the value of APD for calculation of transmembrane voltage in Eq. (4), the restitution curve used in this paper is given below according to cardiac restitution theory:

$$
A P D_{n+1}=f_{\text {restitution }}\left(D I_{n}\right)=315-50 e^{\left(70-D I_{n}\right) / 50}
$$

where the subscript $n+1$ and $n$ denote the $(n+1)^{\text {th }}$ and $n^{\text {th }}$ stimulation cycle, respectively. From the restitution curve, it can be observed that $A P D_{n+1}$ increases with $D I_{n}$ in a nonlinear way. In addition, the cardiac pacing is undatable when $\mathrm{d}\left(A P D_{n+1}\right) / \mathrm{d}\left(D I_{n}\right)>1$, as illustrated in Fig. 1, which happens on the restitution curve determined by Eq. (6) when $D I<70$, or APD $<265$, and BCL=APD+DI $<335$, as marked in Fig. 6. 
With the CAM introduced above, cardiac scenarios such as periodic normal conduction, normal conduction considering scar tissue, spiral wave, and especially alternans can be simulated efficiently in MATLAB, and the case illustrations can be found in [27].

\section{Pacing Protocols}

Downsweep protocol is a widely used pacing method to study cardiac dynamics. Tpically, pacing of the tissue starts from a long $\mathrm{BCL}=\mathrm{BCL}_{0}$ (around $1000 \mathrm{~ms}$ ), and then $\mathrm{BCL}$ is progressively decreased (equivalent BCL is used for constant DI and constant TR pacing). For each specific BCL value, a certain number of stimulations (typically 50) are applied to reach steady state ${ }^{[28][29]}$.

\section{Constant BCL downsweep pacing protocol}

Under constant BCL downsweep pacing, the paced cells are stimulated each time after a constant period. Based on the simulation model introduced above, the values of BCL are set as a series of numbers decreasing from $350 \mathrm{~ms}$ to $300 \mathrm{~ms}$ as shown in Eq. (7).

$$
\begin{aligned}
& B C L(k)=351-k \\
& k=1,2, \mathrm{~L}, 51
\end{aligned}
$$

For each specific constant BCL value in the series, 50 stimulations are applied to ensure the wave reaches steady state.

\section{Constant DI downsweep pacing protocol}

Unlike the constant BCL downsweep pacing protocol, under a constant DI downsweep pacing protocol, the paced cells are stimulated each time after a constant period (i.e., DI) from the instant when their transmembrane voltage drops to the refractory threshold $0.1 \mathrm{~V}$, which is the threshold to distinguish between APD and DI intervals.

In this paper, to test the efficacy of constant DI control on the CAM, the values of DI in control are set as a series of numbers decreasing from $80 \mathrm{~ms}$ to $1 \mathrm{~ms}$ as shown below:

$$
\begin{aligned}
& D I(k)=81-k \\
& k=1,2, \mathrm{~L}, 80
\end{aligned}
$$


For each specific constant DI value in the series, 50 stimulations are applied to test the control efficacy ${ }^{[10][15]}$.

\section{Constant TR downsweep pacing protocol}

Under a constant TR pacing protocol, the paced cells are stimulated each time after a constant period (i.e., TR) from the instant when a T-wave peak on ECG is detected. A peak detection algorithm from [30] is applied in our work to conduct online T-wave peak detection.

The values of TR in control are set as a series of numbers decreasing from $145 \mathrm{~ms}$ to $1 \mathrm{~ms}$, as shown below:

$$
\begin{aligned}
& T R(k)=146-k \\
& k=1,2, \mathrm{~L}, 145
\end{aligned}
$$

For each specific constant TR value in the series, 50 stimulations are applied to test the efficacy of the control ${ }^{[15]}$.

To conduct constant TR pacing, a ECG of the tissue should be recorded. In our CAM-based tissue, with electrode A and electrode B placed out of the tissue (see Fig. 3), the pseudo-ECG can be calculated as:

$$
E C G=\Phi_{e}\left(x_{B}, y_{B}\right)-\Phi_{e}\left(x_{A}, y_{A}\right)
$$

where $\nabla$ denotes gradient operation; $\left(x_{A}, y_{A}\right)$ is the location of electrode $\mathrm{A} ;\left(x_{B}, y_{B}\right)$ is the location of electrode A; and the transmembrane potential $\Phi_{e}$ can be obtained by:

$$
\begin{aligned}
& \left.\Phi_{e}\left(x_{A}, y_{A}\right)=\int(-\nabla V) \mathrm{g} \nabla\left(1 / r_{A}\right)\right) d x d y \\
& \left.\Phi_{e}\left(x_{B}, y_{B}\right)=\int(-\nabla V) \mathrm{g} \nabla\left(1 / r_{B}\right)\right) d x d y
\end{aligned}
$$

where the distances $r_{A}$ and $r_{B}$ in Eq. (11) are provided by:

$$
\begin{aligned}
& r_{A}=\left(\left(x-x_{A}\right)^{2}+\left(y-y_{A}\right)^{2}\right)^{1 / 2} \\
& r_{B}=\left(\left(x-x_{B}\right)^{2}+\left(y-y_{B}\right)^{2}\right)^{1 / 2}
\end{aligned}
$$

where $(x, y)$ is the location of any cell in the tissue.

Pseudo-ECG takes the action potential of all cells in the tissue into consideration; thus, it is a global representation of cardiac dynamics ${ }^{[31]}$. 


\section{SIMULATION RESULTS ON A 50×50 CAM}

This section presents the simulation results under different pacing protocols using a $50 \times 50$ CAM. The central cell located at $(25,25)$ is selected as a representative example to illustrate the pacing stability, and the straight diagonal cable from the paced cell at $(0,0)$ to the corner cell at $(50,50)$ is chosen to show the spatial dynamics. The simulation time step step is $0.1 \mathrm{~ms}$, and the threshold for alternans is set as $1 \mathrm{~ms}$.

\section{Cardiac dynamics under constant BCL downsweep pacing protocol}

Firstly, under constant BCL pacing protocol, the bifurcation diagram of the central cell $(25,25)$ under different BCL values and the spatial alternans along the diagonal cells at a constant $\mathrm{BCL}=320 \mathrm{~ms}$ are shown in Fig. 7.

It can be observed from Fig. 7(a) that alternans occurs at $\mathrm{BCL}_{\mathrm{ONSET}}^{\mathrm{BCL}}=335 \mathrm{~ms}$, which is the same as the value calculated from the restitution curve in Eq. (6), and conduction block happens at BCL ${ }_{\mathrm{CB}}^{\mathrm{BCL}}=311 \mathrm{~ms}$. Spatial concordant alternans (SCA) occurs along the diagonal cable as shown in Fig. 7(b) when BCL=320 ms, which also indicates SCA in the 2-D tissue.

Illustrations of normal response occurs at $\mathrm{BCL}=340 \mathrm{~ms}$ and alternans occurs at $\mathrm{BCL}=320 \mathrm{~ms}$ generated by a $200 \times 200$ CAM are shown in Fig. 8 . From Fig.8 one can see that the waves have the same length (yellow color) under normal response but oscillate between small and large values under alternans.

Thus, the CAM provides an intuitive visual illustration of cardiac dynamics. Also, transmembrane voltage of the cell $(25,25)$ under constant $\mathrm{BCL}=340 \mathrm{~ms}$ and $\mathrm{BCL}=320 \mathrm{~ms}$ are shown in Fig. 9.

From Fig. 9(a), we can also see that when BCL=340 ms, the APD remains constant, thus it is normal response. However, the APD under BCL=320 ms shown in Fig. 9(b) alternates.

\section{Cardiac dynamics under constant DI downsweep pacing protocol}

Secondly, under the constant DI pacing protocol, the results of the same central cell and diagonal cable as that in the constant BCL test are shown in Fig. 10. Here, the equivalent BCL is calculated by $f_{\text {resitution }}(D I)+D I$. Also, because $\mathrm{BCL}=f_{\text {resitution }}(1)+1 \approx 117 \mathrm{~ms}$ when $\mathrm{DI}=1 \mathrm{~ms}$, the limit of BCL under constant DI control pacing is given as $\mathrm{BCL}_{\mathrm{LIMIT}}^{\mathrm{DI}}=117 \mathrm{~ms}$. 
From the results in Fig. 10, there is no bifurcation, and the wave propagation can always be stable under constant DI protocol even when DI is $1 \mathrm{~ms}$ with a corresponding equivalent $\mathrm{BCL}$ less than $120 \mathrm{~ms}$.

To better show the dynamic details, transmembrane voltage of cell $(25,25)$ under constant DI=60 ms (equivalent $\mathrm{BCL}=314 \mathrm{~ms}$ ), and $\mathrm{DI}=20$ (equivalent $\mathrm{BCL}=199 \mathrm{~ms}$ ), are shown in Fig. 11 . From Fig. 11, it can be observed that no matter under relative large or small equivalent BCL, e.g. under $\mathrm{DI}=60 \mathrm{~ms}$ or $\mathrm{DI}=20 \mathrm{~ms}$, pacing of the cell $(25,25)$ can always be stablilized.

Thus, simulation results validate the high efficacy of constant DI pacing on the 2-D CAM-based tissue.

\section{Cardiac dynamics under constant TR downsweep pacing protocol}

Thirdly, results under the constant TR pacing protocol are shown in Fig. 12. In the simulation, two electrodes are placed at $(0,0)$ and $(51,51)$ to generate the ECG.

From Fig. 12 we can see that the pacing is stable under constant TR pacing when TR is bigger than $26 \mathrm{~ms}$ with an equivalent BCL bigger than $117 \mathrm{~ms}$. Also, conduction block occers at $\mathrm{BCL}_{\mathrm{CB}}^{\mathrm{TR}}$ $=117 \mathrm{~ms}$, and no alternans is observed in the whole process.

To illustrate detailed dynamics of the tissue under constant TR control, ECGs of the tissue and transmembrane voltage of the cell $(25,25)$ under $\mathrm{TR}=118 \mathrm{~ms}$ (equivalent $\mathrm{BCL}=313 \mathrm{~ms}$ ) and $\mathrm{TR}=61 \mathrm{~ms}$ (equivalent $\mathrm{BCL}=200 \mathrm{~ms}$ ) are shown in Fig. 13 and Fig. 14, respectively.

From Fig. 13 and Fig. 14, no matter under relative large or small equivalent BCL, e.g. under $\mathrm{TR}=118 \mathrm{~ms}$ or $\mathrm{TR}=61 \mathrm{~ms}$, both $\mathrm{ECG}$ of the tissue and pacing of the cell $(25,25)$ can always be stablilized, which indicates normal response of the whole tissue.

Thus, the simulation results validate that constant TR control can achieve the same performance as constant $\mathrm{DI}$ control until conduction block happens, and $\mathrm{BCL}_{\mathrm{CB}}^{\mathrm{TR}}=\mathrm{BCL}_{\mathrm{LIMIT}}^{\mathrm{DI}}$.

\section{DYNAMIC RESPONSE UNDER DIFFERENT DOWNSWEEP PROTOCOLS WITH DIFFERENT DECREASE STEPS FOR DIFFERENT MODEL SIZE}


In the last section, the efficacy of both constant DI and constant TR approaches were validated on a 50×50 model using a downsweep protocol where the DI and TR decrease by $1 \mathrm{~ms}$ each step. The results show that both constant DI and constant TR control approaches could help to significantly decrease the equivalent BCL at which unstable dynamics occurs. More specifically, both approaches are fully effective in controlling alternans until an equivalent BCL is smaller than $\mathrm{BCL}_{\mathrm{LIMIT}}^{\mathrm{DI}}$, which is the smallest BCL that can be given by restitution curve.

However, the results in [17] show that both constant DI and constant TR pacing protocols have limited effect on alternans preventation when a $5 \mathrm{~ms}$ decrease step is adopted in the downsweep protocol, and both alternans and conduction block will occur.

Thus, to fully study efficacy of the two control methods, in this section, dynamic responses of the system under the three pacing protocols, e.g. constant BCL, constant DI, and constant TR, using downsweep protocols with different decrease steps are studied along with a consideration of model size.

Firstly, downsweep protocols with different "decrease steps" $\Delta$ of $1 \mathrm{~ms}, 5 \mathrm{~ms}$, and $10 \mathrm{~ms}$ are applied in constant BCL, constant DI, and constant TR pacing protocols, as shown in Eq. (13). For example, when $\Delta=5 \mathrm{~ms}$, we can get $\operatorname{DI}(k)=(80-5(k-1))=85-5 k=\{80,75,70, \ldots, 10,5\}$. Secondly, size of the square model was varied from $4 \times 4$ size to $50 \times 50$ size.

$$
\begin{aligned}
& B C L(k)=(350-\Delta \cdot(k-1)) \in[300,350] m s \\
& D I(k)=(80-\Delta \cdot(k-1)) \quad \in[1,80] m s \\
& T R(k)=(145-\Delta \cdot(k-1)) \in[1,145] m s \\
& k=1,2,3, \mathrm{~L} \\
& \Delta \in\{1,5,10\} m s
\end{aligned}
$$

where $\Delta$ is the decrease step of downsweep protocols.

Performance of the three pacing protocols was tested and results are shown in Fig. 15-17. From simulation results, system dynamics remain the same when the model size is bigger than $20 \times 20$, thus only performance on tissues with a size less than 20 is given in $\mathrm{BCL}_{\text {ONSET }}$ comparison. In addition, the $\mathrm{BCL}_{\mathrm{CB}}$ does not change with model size and is plotted in the histogram.

From the results, it can be observed that: 
(1) For constant BCL pacing protocol, the simulation results, including $\mathrm{BCL}_{\mathrm{ONSET}}$ and $\mathrm{BCL}_{\mathrm{CB}}$ remain the same independend of the model size and $\Delta$.

(2) Both constant DI and constant TR pacing protocols are effective to prevent alternans compared with constant BCL pacing, and both protocols stablize cardiac response till the minimum equivalent BCL, e.g. $\mathrm{BCL}_{\mathrm{LIMIT}}^{\mathrm{DI}}$, for $\Delta=1 \mathrm{~ms}$. However, their efficacy decays with the increase of $\Delta$ in the downsweep protocol.

(3) For $\Delta>1 \mathrm{~ms}$, i.e. $5 \mathrm{~ms}$ and $10 \mathrm{~ms}$, the constant TR control is generally more effective than constant DI control.

In comparison, paper [17] used a fixed decrease step $\Delta=5 \mathrm{~ms}$ and presented that both constant DI and constant TR will cause alternans and conduction block when values of DI and TR are small. This is the same as what we observed in the CAM when decrease step $\Delta$ is set as $5 \mathrm{~ms}$ or $10 \mathrm{~ms}$ in the downsweep protocol. However, the effect of different decrease steps on control efficacy has not been studied and is first investigated in this paper. As observed from the numerical simulation, both constant DI and constant TR approaches are fully effective if the decrease step $\Delta$ in downsweep protocols are small enough.

\section{CONCLUSION AND DISCUSSION}

Based on a 2-D CAM, the efficacy of constant DI and constant TR pacing protocols on alternans preventation or elimination are studied in this paper by numerical simulation. Unlike the existing simulation work focusing on 0-D or 1-D tissue, the work in this paper is done on a 2-D model. Although less accurate than real heart experiments, CAM provides an economical and efficient approach for cardiac dynamic research and establishes a basis for both higher dimensional cardiac simulation and alternans prevention in heart tissue.

Simulation experiments in this paper validate that:

(1) CAM provides high efficiency and intuitive visual illustration for cardiac electrical wave propagation simulation.

(2) Both constant DI and constant TR protocols are effective to resolve cardiac alternans. However, their efficacy depends on the adopted decrease step in downsweep protocols, and a small decrease step could improve their performance.

In the meanwhile, there are some limitations of this study: 
(1) The employed CAM uses simple rules to simulate wave propagation, and the accuracy is not as high as the traditional full physiological model. The rules can be improved to keep a better balance between efficiency and accuracy.

(2) The intracellular calcium cycling which is important for alternans development is not considered.

Future work can be done on the following aspects:

(1) Using a 3-D CAM to simulate caidiac wave propagation and study the efficacy of different control approaches.

(2) Improving the rules of CAM so that more details can be included without a significant sacrifice of computation efficiency.

(3) Study the essential mechanism in the dependence of control efficacy and decrease step in downsweep protocols.

(4) Conduct tests on well-developed two-dimensional full physiological simulation models or ex vivo whole animal hearts to validate the results obtained from the CAM-based simulation in this paper.

\section{CONFLICT OF INTEREST}

The authors declare that they have no conflict of interest.

\section{FUNDING}

This work was supported in part by the National Science Foundation under grant number 1661615 and grant number 1659502.

\section{DATA AVAILABILITY STATEMENT}

The data that support the findings of this study are available from the corresponding author upon reasonable request. 


\section{REFERENCES}

[1] T. You, C. Luo, K. Zhang, and H. Zhang, "Electrophysiological mechanisms underlying t-wave alternans and their role in arrhythmogenesis," Front. Physiol. 12, 235 (2021).

[2] R. Mehra, "Global public health problem of sudden cardiac death,” Journal of electro cardiology. 40(6), S118S122 (2007).

[3] X. Zhao, and E. G. Tolkacheva, "A reduced order model for spatiotemporal dynamics and control of cardiac alternans,” Dynamic Systems and Control Conference. 51890 (2018).

[4] K. Hall, D. J. Christini, M. Tremblay, J. J. Collins, L. Glass, and J. Billette, "Dynamic control of cardiac alternans," Physical Review Letters. 78(23), 4518 (1997).

[5] S. Sridhar, D. M. Le, Y. C. Mi, S. Sinha, P. Y. Lai, and C. K. Chan, "Suppression of cardiac alternans by alternating-period-feedback stimulations,” Phys. Rev. E 87(4), 042712 (2013).

[6] D. J. Christini, M. L. Riccio, C. A. Culianu, J. J. Fox, A. Karma, and R. F. Gilmour Jr, “Control of electrical alternans in canine cardiac Purkinje fibers,” Physical review letters. 96(10), 104101 (2006).

[7] S. D. McIntyre, V. Kakade, Y. Mori, and E. G. Tolkacheva, "Heart rate variability and alternans formation in the heart: The role of feedback in cardiac dynamics," Journal of theoretical biology. 350, 90-97 (2014).

[8] B. Echebarria and A. Karma, "Spatiotemporal control of cardiac alternans," Chaos 12, 923-930 (2002).

[9] U. B. Kanu, S. Iravanian, R. F. Gilmour, and D. J. Christini, "Control of action potential duration alternans in canine cardiac ventricular tissue,” IEEE Transactions on Biomedical Engineering. 58(4), 894-904 (2010). [10] P. Parthiban, "Effect of constant-DI pacing on single cell cardiac dynamics," PhD diss., University of Minnesota, (2020).

[11] E. M. Cherry, "Distinguishing mechanisms for alternans in cardiac cells using constant-diastolic-interval pacing," Chaos 27, 093902 (2017).

[12] S. Zlochiver, C. Johnson, and E. G. Tolkacheva, "Constant DI pacing suppresses cardiac alternans formation in numerical cable models," Chaos 27, 093903 (2017).

[13] R. Wu, A. Patwardhan, "Mechanism of repolarization alternans has restitution of action potential duration dependent and independent components," Journal of cardiovascular electrophysiology. 17(1), 87-93 (2006). [14] Niels F. Otani, "Theory of the development of alternans in the heart during controlled diastolic interval pacing," Chaos 27, 093935 (2017).

[15] K. Kulkarni, S. W. Lee, R. Kluck, and E. G. Tolkacheva, (2018). "Real-time closed loop diastolic interval control prevents cardiac alternans in isolated whole rabbit hearts," Annals of biomedical engineering. 46(4), 555-566 (2018).

[16] R. L. Verrier, M. Malik, "Electrophysiology of T-wave alternans: mechanisms and pharmacologic influences," Journal of electrocardiology. 46(6), 580-584 (2013).

[17] S. Thakare, J. Mathew, S. Zlochiver, X. Zhao, and E. G. Tolkacheva, "Global vs local control of cardiac alternans in a 1D numerical model of human ventricular tissue," Chaos 30, 083123 (2020). 
[18] K. Kulkarni, R. D. Walton, A. A. Armoundas, and Tolkacheva, E. G, “Clinical potential of beat-to-beat diastolic interval control in preventing cardiac arrhythmias," Journal of the American Heart Association. 10(11), e020750 (2021).

[19] J. S. McCaskill, N. H Packard, “Analysing emergent dynamics of evolving computation in 2D cellular automata," International Conference on Theory and Practice of Natural Computing. Springer. Cham. 3-40 (2019).

[20] R. S. Campos, R. M. Amorim, et al, “3D heart modeling with cellular automata, mass-spring system and CUDA,” International Conference on Parallel Computing Technologies. Springer, Berlin, Heidelberg. 296-309 (2013).

[21] S. A. Avdeev, N. M Bogatov. "Simulation of electrochemical processes in cardiac tissue based on cellular automaton,” IOP Conference Series: Materials Science and Engineering. 66(1), 012019 (2014).

[22] M. S. Khan, S. Yousuf, “A cardiac electrical activity model based on a cellular automata system in comparison with neural network model,” Pak J Pharm Sci. 29(2), 579-84 (2016).

[23] Y. T. Lin, E. T. Chang, J. Eatock, T. Galla, and R. H. Clayton, "Mechanisms of stochastic onset and termination of atrial fibrillation episodes: Insights using a cellular automaton model,” arXiv preprint. 1612.03403 (2016).

[24] R. H. Mitchell, A. H. Bailey, and J. Anderson, “Cellular automaton model of ventricular fibrillation," IEEE transactions on biomedical engineering. 39(3), 253-259 (1992).

[25] A. B. Feldman, Y. B. Chernyak, and R. J. Cohen, "Cellular automata model of cardiac excitation waves," Herzschr Elektrophys. 10(2), 92-104 (1999).

[26] F. Pourhasanzade, S. H. Sabzpoushan, "A new cellular automata model of cardiac action potential propagation based on summation of excited neighbors," World Academy of Science, Engineering and Technology. 44, 917-921 (2010).

[27] D. Gallenberger, M. Xiong, T. Z. Zhuang, K. Sun, E. G. Tolkacheva, and X. Zhao, “A cellular automata model for dynamics and control of cardiac arrhythmias," Dynamic Systems and Control Conference. 84270, V001T08A003 (2020).

[28] S. S. Kalb, H. M. Dobrovolny, E. G. Tolkacheva, et al, "The restitution portrait: A new method for investigating rate-dependent restitution," Journal of cardiovascular electrophysiology. 15(6), 698-709 (2004).

[29] X. Zhao, "Indeterminacy of spatiotemporal cardiac alternans," Physical Review E 78(1), 011902 (2008).

[30] F. Scholkmann, J. Boss, and M. Wolf. "An efficient algorithm for automatic peak detection in noisy periodic and quasi-periodic signals," Algorithms. 5(4), 588-603 (2012).

[31] M. Dupraz, S. Filippi, A. Gizzi, A. Quarteroni, and R. Ruiz-Baier, "Finite element and finite volume-element simulation of pseudo-ECGs and cardiac alternans," Mathematical Methods in the Applied Sciences. 38(6), 1046-

1058 (2015). 


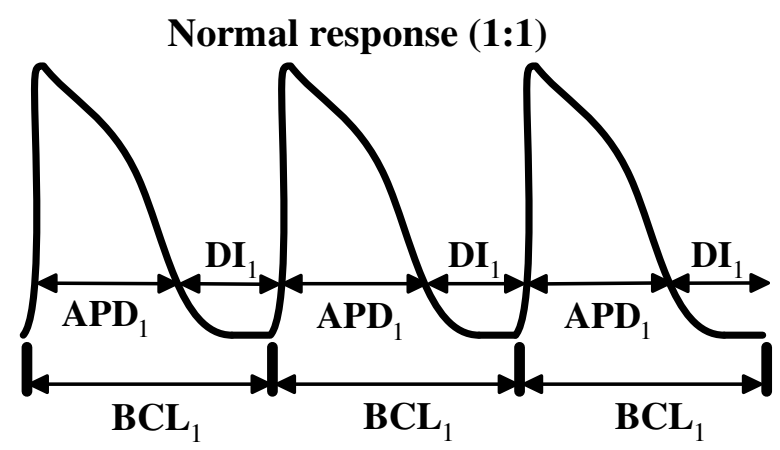

(a)

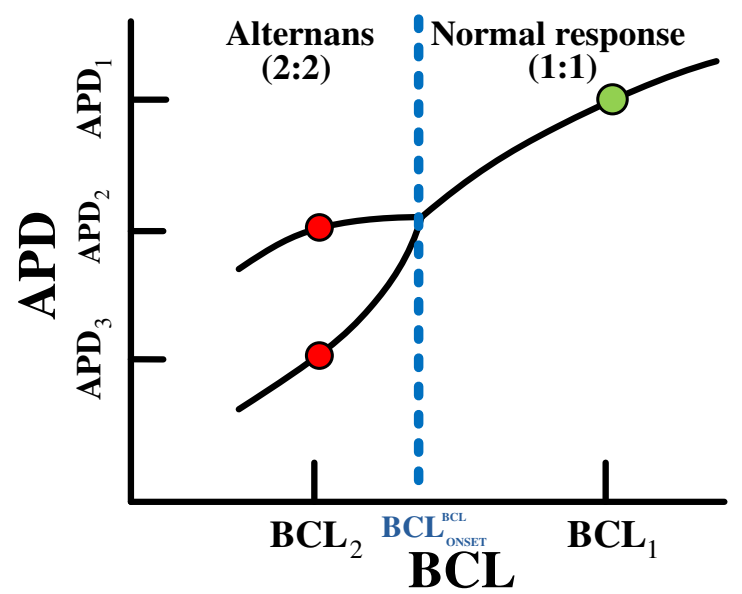

(c)

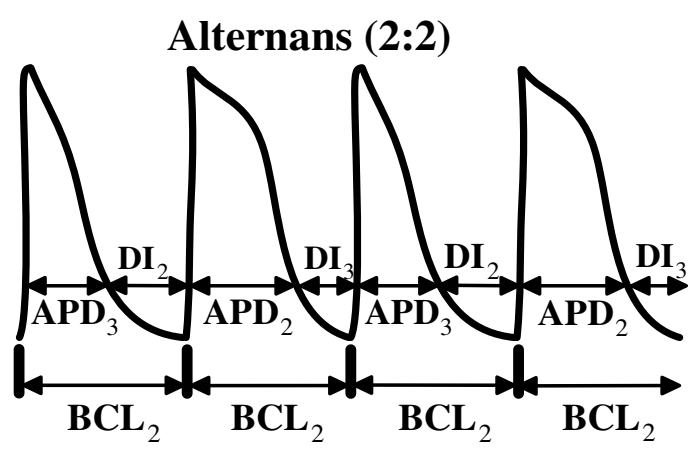

(b)

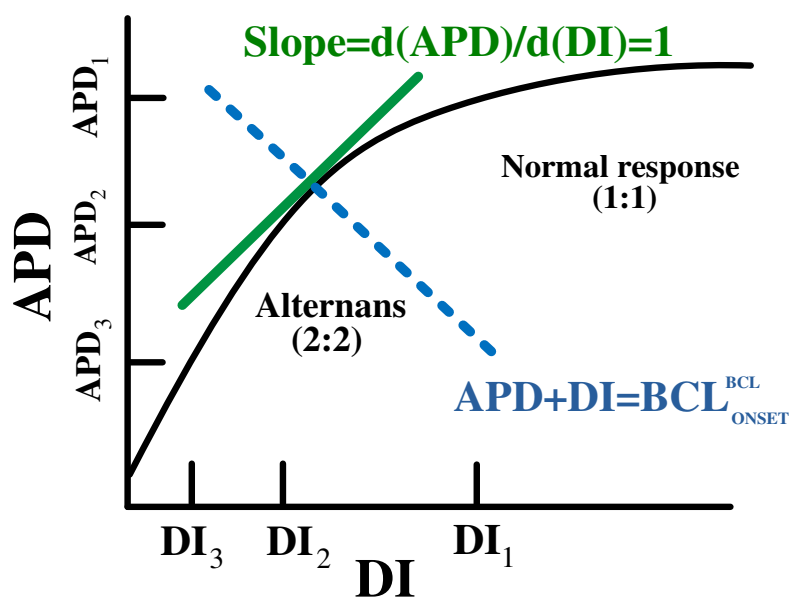

(d)

FIG. 1. Illustration of APD bifurcation under constant BCL pacing. (1) During normal response, APDs in each cycle remains the same. (2) During alternans, APD alternates between two different values. (3) Under constant BCL pacing, normal response occurs when $B C L \geq B C L_{\text {ONSET }}^{\mathrm{BCL}}$ and alternans occurs when $B C L<B C L_{\text {ONSET }}^{\mathrm{BCL}}$. (4) Under constant $\mathrm{BCL}$ pacing, at the point where slope of the restitution curve is 1 , we have $\mathrm{APD}+\mathrm{DI}=\mathrm{BCL}_{\mathrm{ONSE}}^{\mathrm{BCL}}$, and when the slope is bigger than 1, $\mathrm{APD}+\mathrm{DI}>\mathrm{BCL}_{\mathrm{ONSET}}^{\mathrm{BC}}$ and the pacing is normal, also, when slope is smaller than 1 , then $\mathrm{APD}+\mathrm{DI}<\mathrm{BCL} \mathrm{L}_{\mathrm{ONSET}}^{\mathrm{BCL}}$ and alternans occurs. 


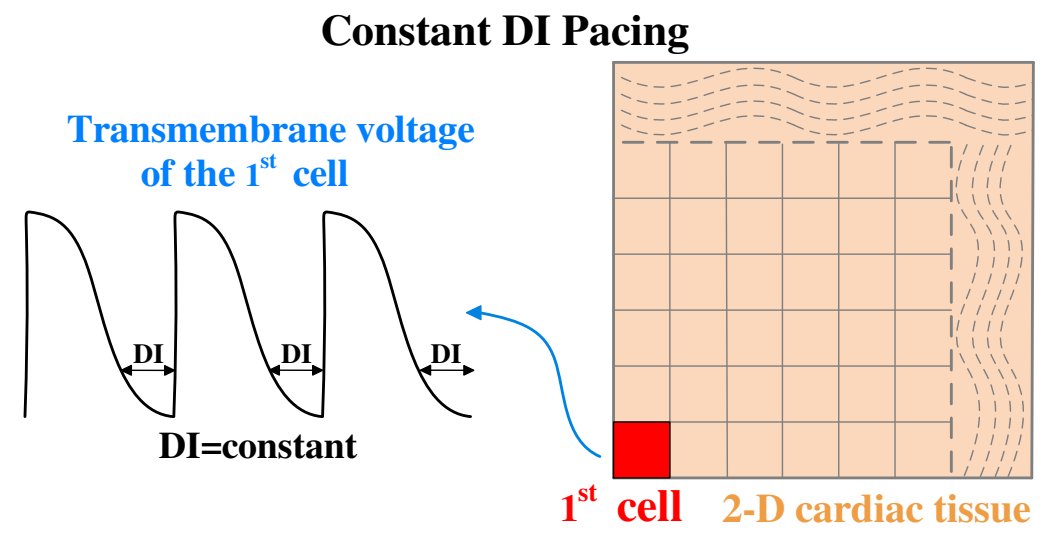

FIG. 2. Illustration of Constant DI pacing. In a 2-D tissue represented by the big yellow square, based on measured transmembrane voltage of the $1^{\text {st }}$ cell which is marked in red, the tissue is paced after a preset duration when the transmembrane voltage drops below the threshold of DI.

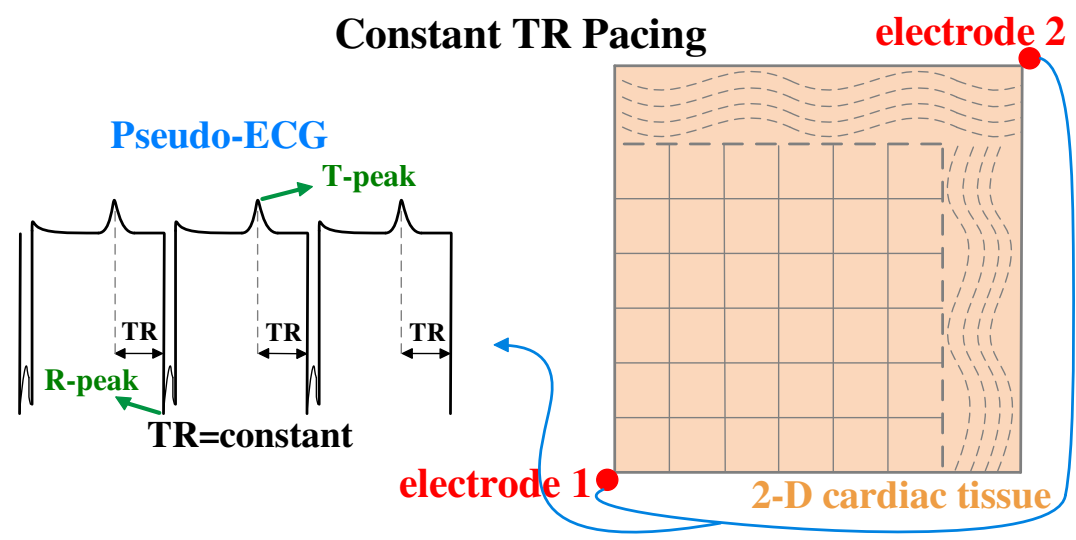

FIG. 3. Illustration of Constant TR pacing. In a 2-D tissue represented by the big yellow square, based on ECG of the whole tissue which is measured by two electrodes, the tissue is paced after a preset duration when the T-peak is detected.

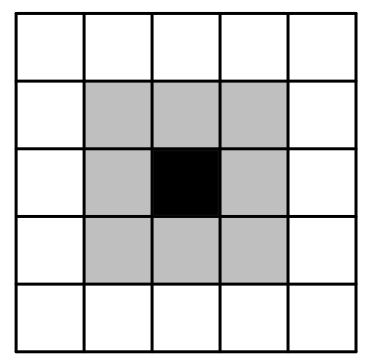

(a)

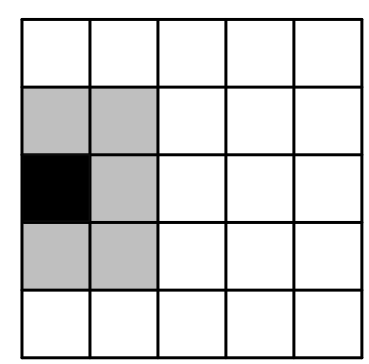

(b)

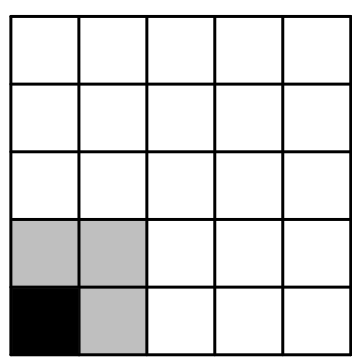

(c)

FIG. 4. Neighboring cells of a cell in a CAM. A black square represents any cell in the tissue, the gray squares are neighboring cells of the black cell. (a) A inner cell has eight neighboring cells. (b) A boundary cell has five neighboring cells. (c) A corner cell has three neighboring cells. During the wave propagation, to excite a black cell which is in relative refractory or resting state, at least three of its neighbors must be in excitation or absolute refractory state. 


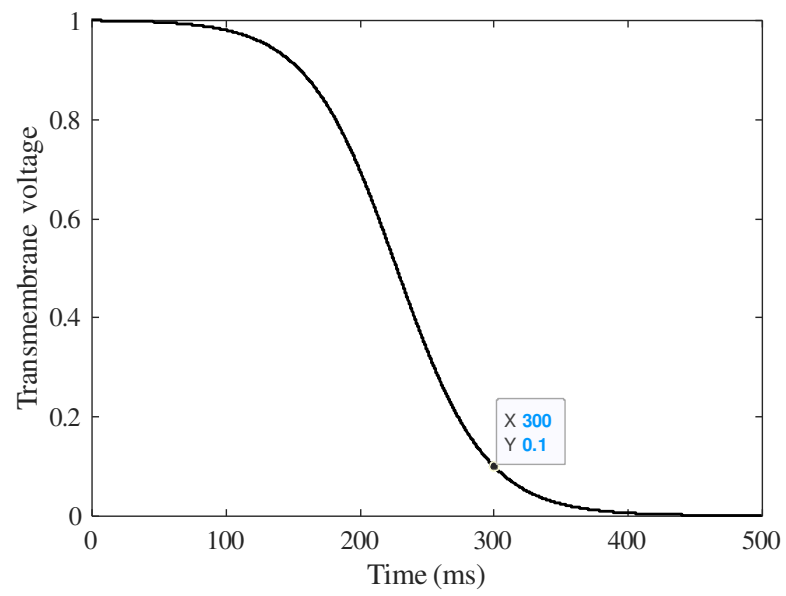

FIG. 5. Transmembrane voltage waveform when $\mathrm{APD}=300 \mathrm{~ms}$. When a cell is excited, its transmembrane voltage becomes $1.0 \mathrm{~V}$ and then drops according to the voltage waveform. Its voltage will become 0.1 when the elapsed time is equal to $\mathrm{APD}=300 \mathrm{~ms}$.

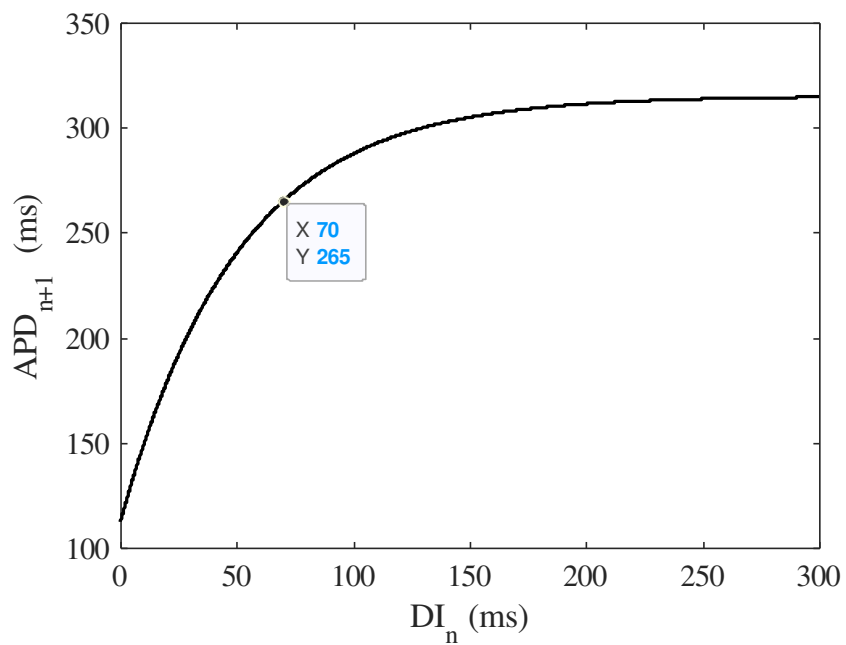

FIG. 6. Restitution curve in the CAM. The curve slope is 1 at the marked point $(70,265)$. Thus, under constant BCL pacing, alternans occurs when $\mathrm{BCL}<(70+265) \mathrm{ms}=335 \mathrm{~ms}$. 


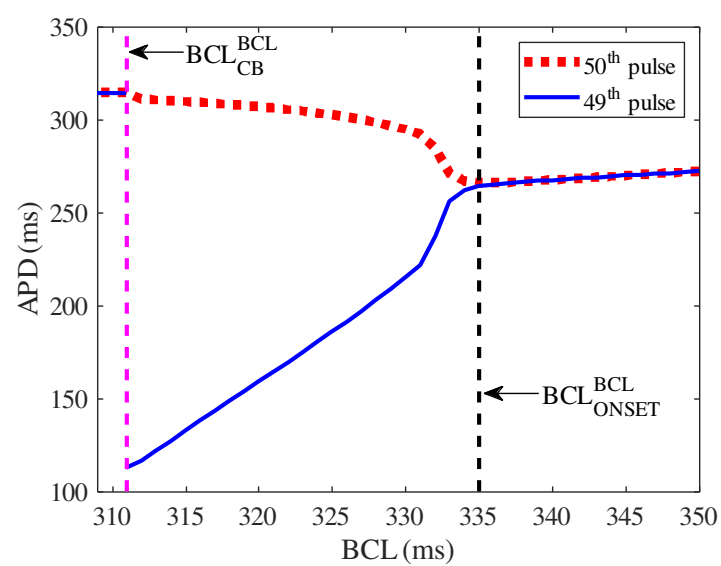

(a)

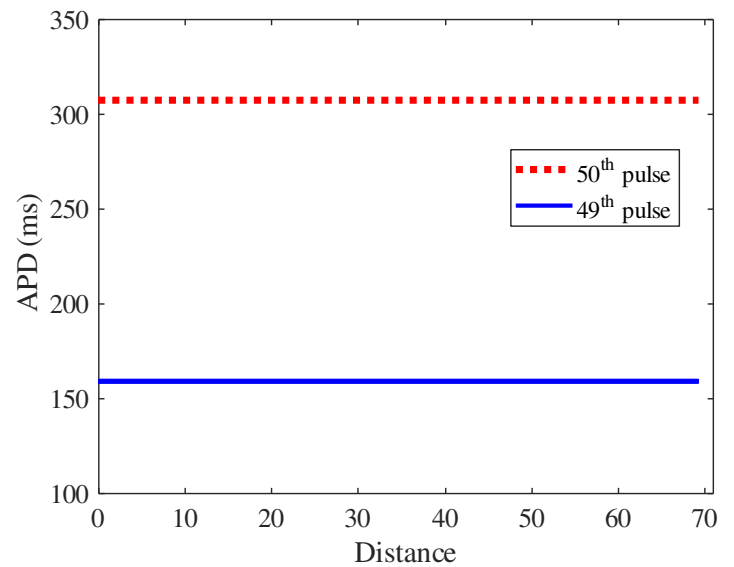

(b)

FIG. 7. Results of 2-D 50×50 model paced with constant BCL pacing protocol. (a) Bifurcation diagram at $(25,25)$. $\mathrm{BCL}_{\mathrm{ONSET}}^{\mathrm{BCL}}=335 \mathrm{~ms}$ is marker with vertical black dashed line; $\mathrm{BCL}_{\mathrm{CB}}^{\mathrm{BCL}}=311 \mathrm{~ms}$ is marker with vertical pink dashed line. APD of cell $(25,25)$ equals to $267.4 \mathrm{~ms}$ when $\mathrm{BCL}=340$, and alternans between $159 \mathrm{~ms}$ and $307 \mathrm{~ms}$ when $\mathrm{BCL}=320 \mathrm{~ms}$. (b) APD of the last two stimulation pulses along the straight diagonal cable from $(1,1)$ to $(50,50)$ at a $\mathrm{BCL}=320 \mathrm{~ms}$, where $x$ axis is the distance of each cell to point $(1,1)$, and alternans of APD between $159 \mathrm{~ms}$ and 307 ms can also be observed. 

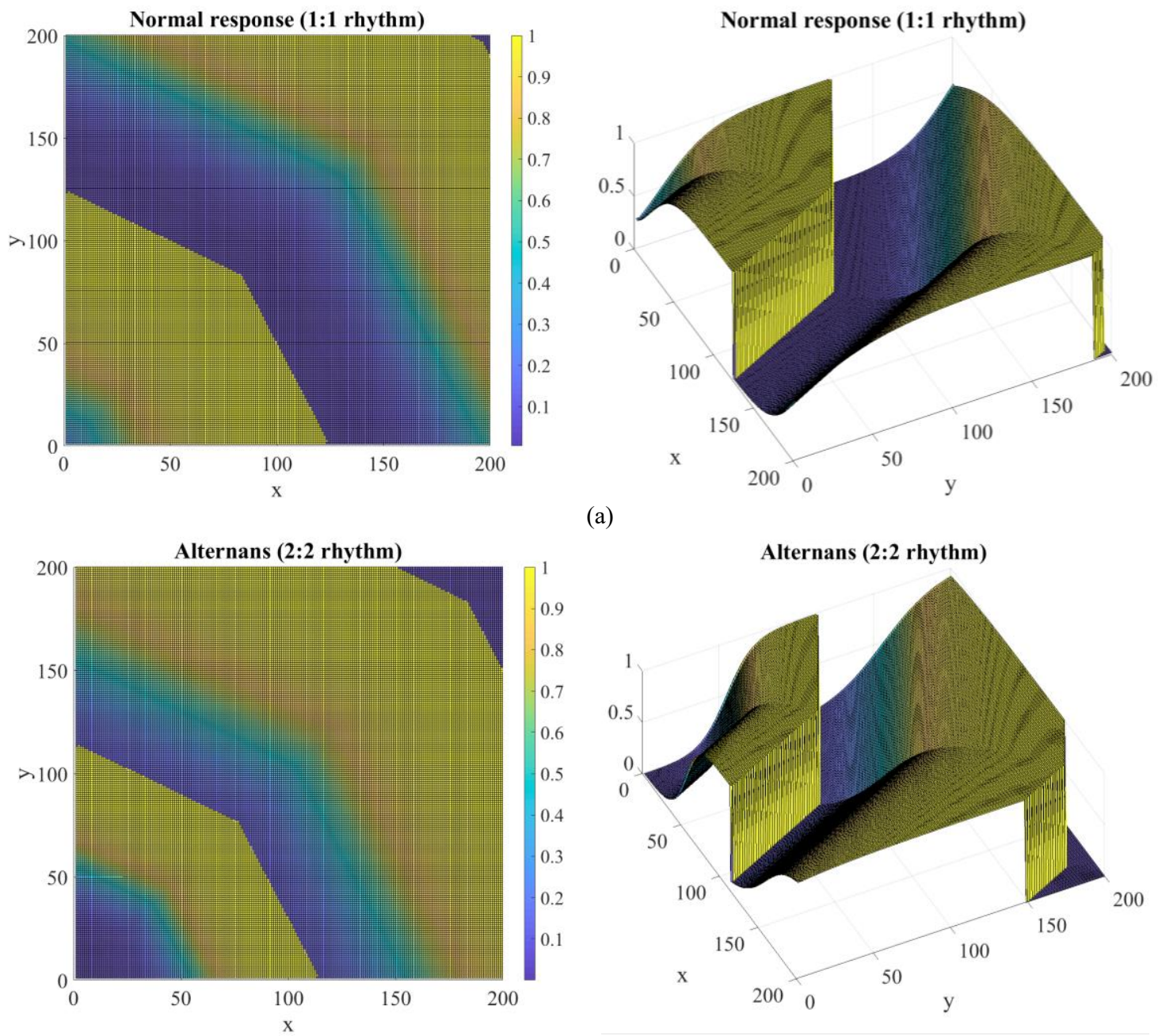

(a)

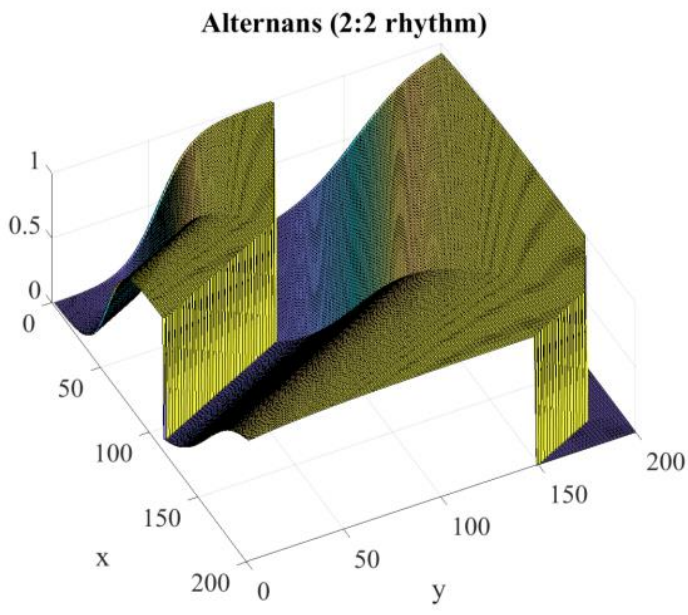

(b)

FIG. 8. Illustration of normal response and alternans in the CAM. Transmembrane voltage is represented by different colors as shown in the color bar. The left-hand-side pictures are in 2-D view and right-hand-side pictures are in 3-D view. (a) When $B C L=340 \mathrm{~ms}$, caidiac waves have the same wave length and manifest 1:1 rhythm. (b) When $B C L=320 \mathrm{~ms}$, caidiac waves have different wave lengthes and manifest 2:2 rhythm. 


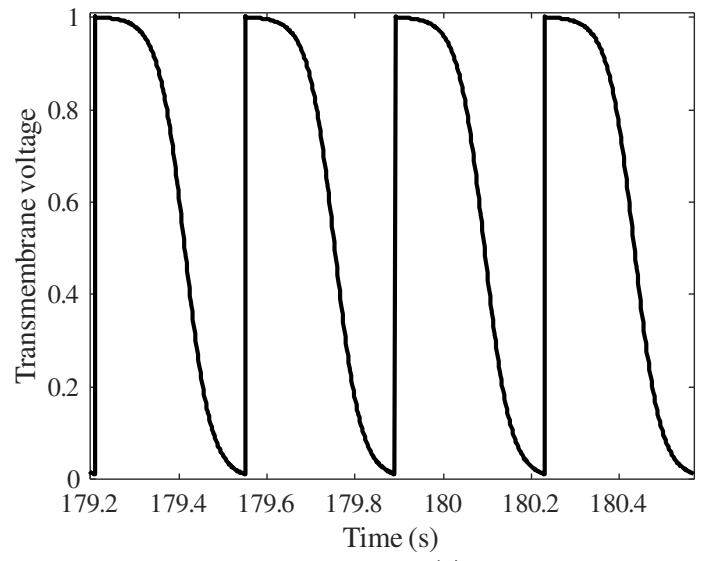

(a)

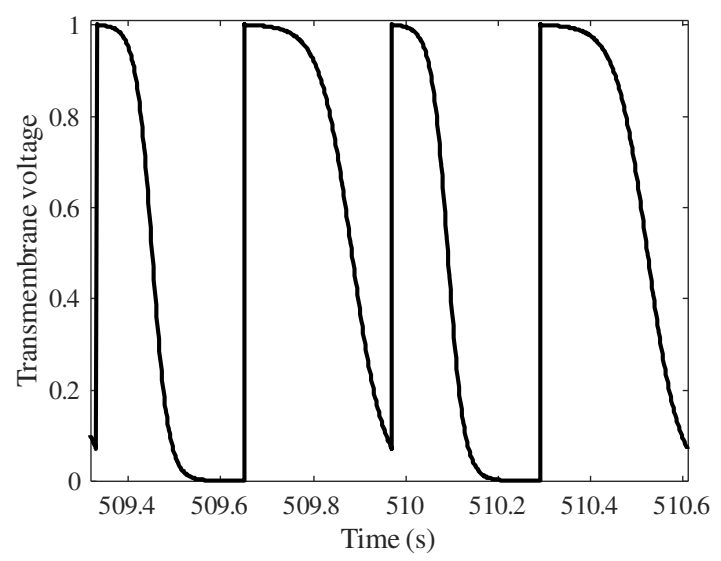

(b)

FIG. 9. Transmembrane voltage of cell $(25,25)$ under constant BCL pacing protocol. (a) When BCL=340 ms, APD of the cell $(25,25)$ is constant. (b) When BCL $=320 \mathrm{~ms}$, APD of the cell $(25,25)$ alternantes.

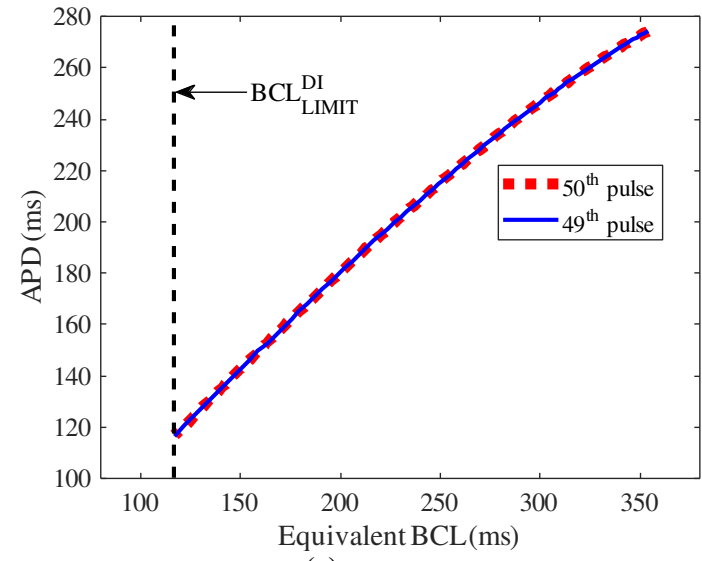

(a)

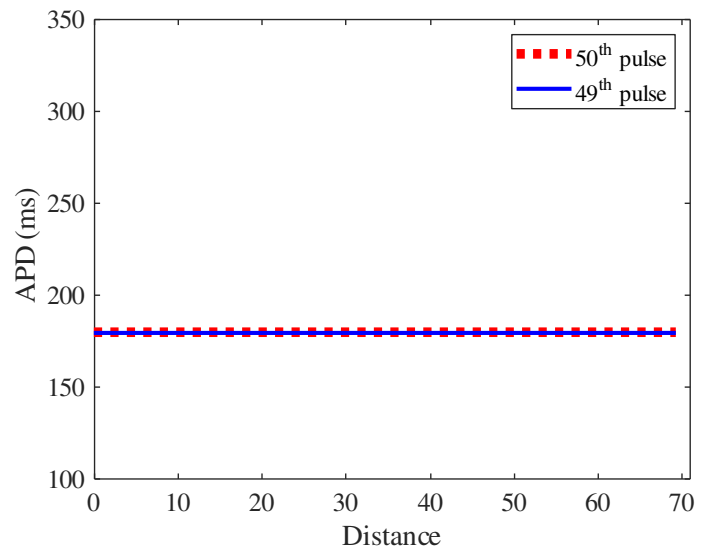

(b)

FIG. 10. Results of 2-D 50×50 model paced with constant DI protocol. (a) Bifurcation diagram at cell $(25,25)$. BCL $\underset{\text { LIMIT }}{\text { DI }}=117 \mathrm{~ms}$ is marker with vertical black dashed line. The pacing maintains as 1:1 rhythm under constant DI control. (b) APD of the last two stimulation pulses along the straight diagonal cable from $(1,1)$ to $(50,50)$ at $\mathrm{DI}=20$ ms, where $x$ axis is the distance of each diagonal cell to point $(1,1)$. 


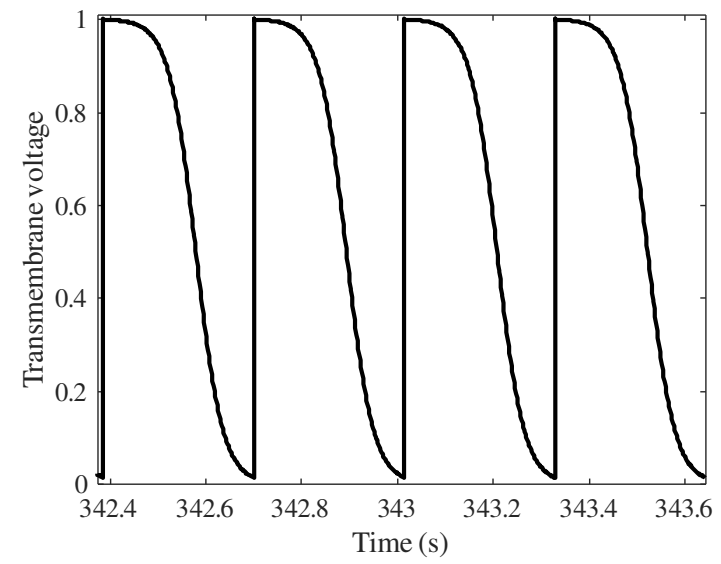

(a)

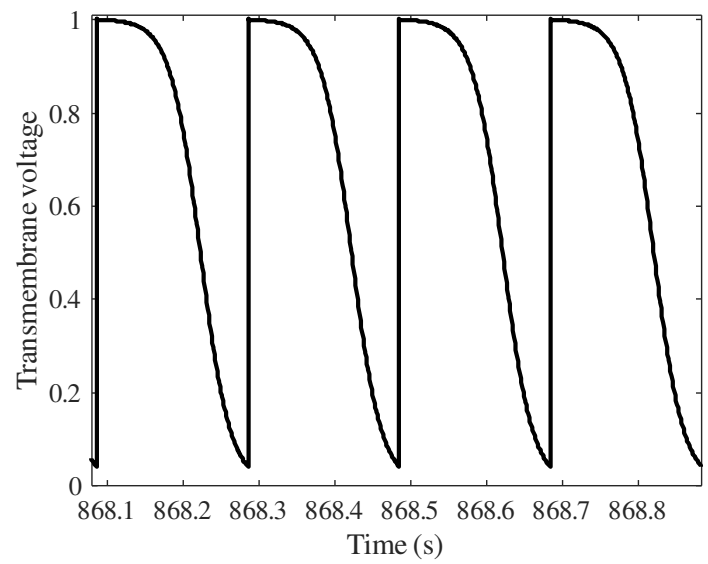

(b)

FIG. 11. Transmembrane voltage of cell $(25,25)$ under constant DI pacing protocol. (a) When DI=60 ms, the equivalent BCL is $314 \mathrm{~ms}$, and APD of the cell $(25,25)$ remains constant. (b) When DI=20 ms, the equivalent BCL is $199 \mathrm{~ms}$, and APD of the cell $(25,25)$ also remains constant.

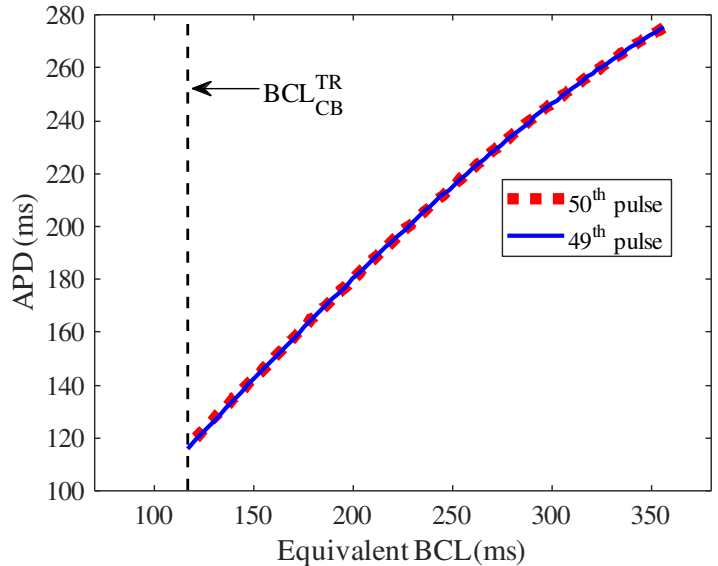

(a)

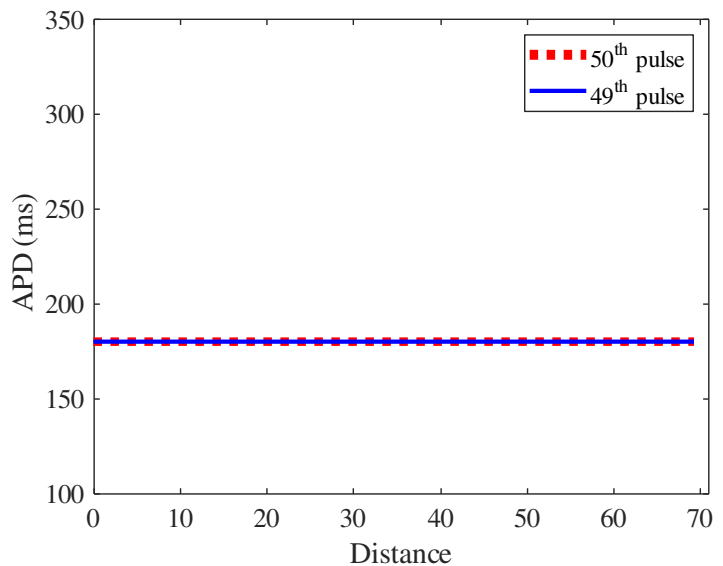

(b)

FIG. 12. Results of 2-D 50×50 model paced with constant TR protocol. (a) Bifurcation diagram at cell $(25,25)$. $\mathrm{BCL}_{\mathrm{CB}}^{\mathrm{TR}}=117 \mathrm{~ms}$ is marker with vertical black dashed line. The pacing maintains as 1:1 rhythm under constant TR control until reaching $\mathrm{BCL}_{\mathrm{CB}}^{\mathrm{TR}}$. (b) APD of the last two stimulation pulses along the straight diagonal cable from $(1,1)$ to $(50,50)$ at TR=61 ms, where $x$ axis is the distance of each diagonal cell to point $(1,1)$. 


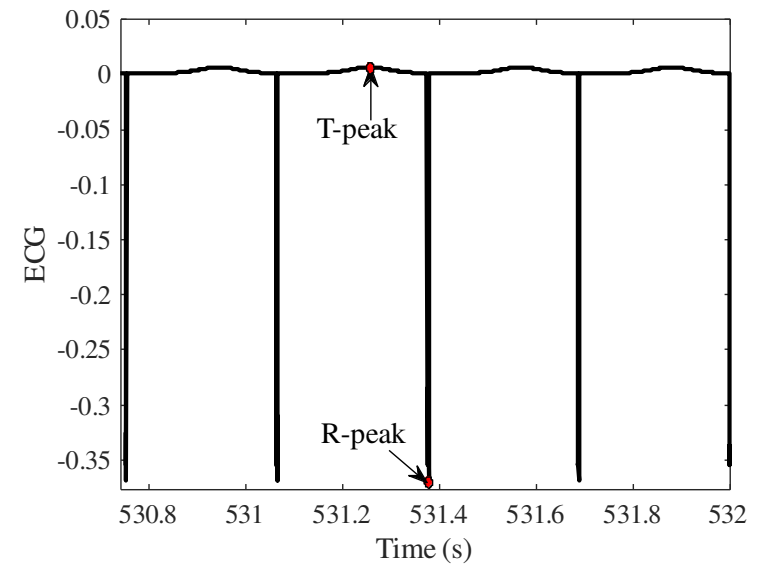

(a)

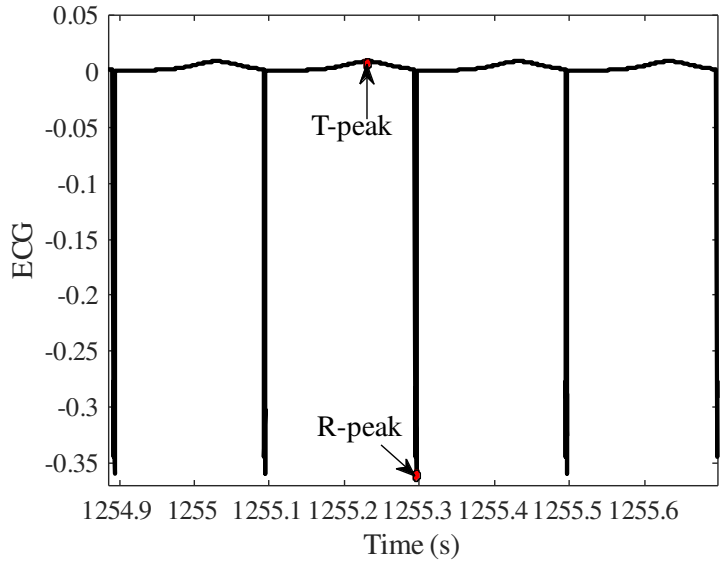

(b)

FIG. 13. ECG of the tissue under constant TR pacing protocol. T-peaks and R-peaks are marked as red points. ECG of every cycle having the same shape indicates 1:1 pacing rythym. (a) When TR=118 ms, the equivalent BCL is 313 $\mathrm{ms}$, and the pacing is stable. (b) When TR=61 ms, the equivalent BCL is $200 \mathrm{~ms}$, and the pacing is also stable.

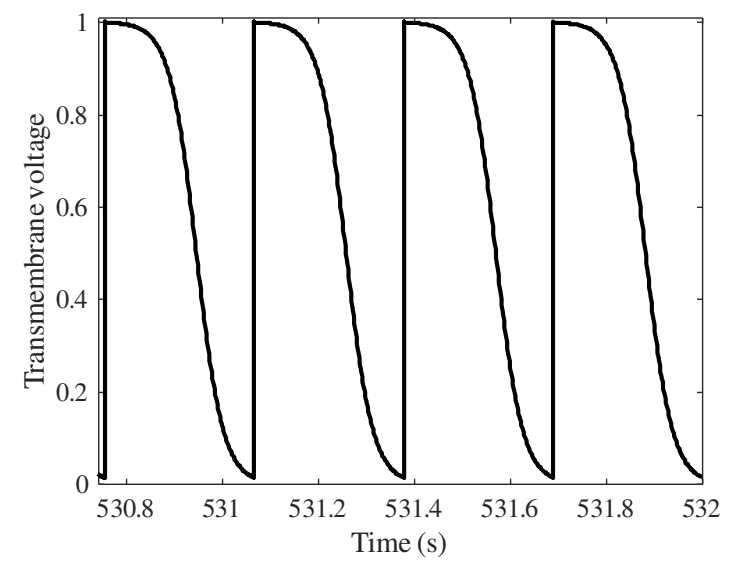

(a)

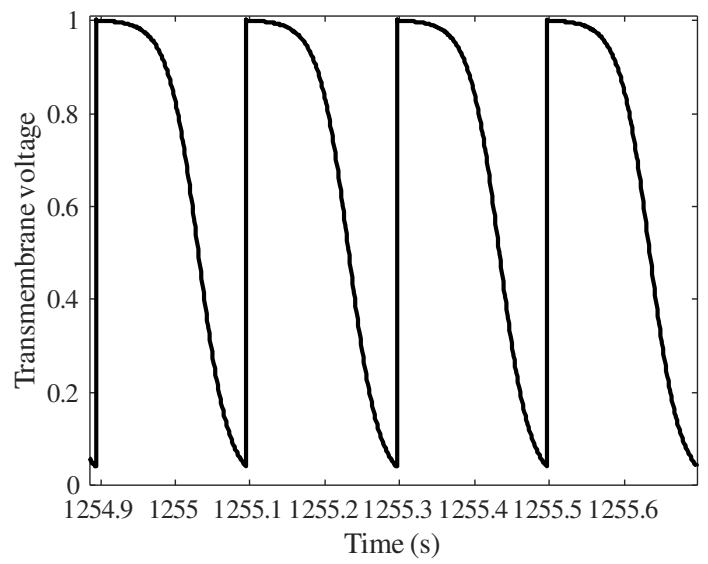

(b)

FIG. 14. Transmembrane voltage of cell $(25,25)$ under constant TR pacing protocol. (a) When TR=118 ms, APD of the cell $(25,25)$ remains constant. (b) When TR=61 ms, APD of the cell $(25,25)$ also remains constant. 


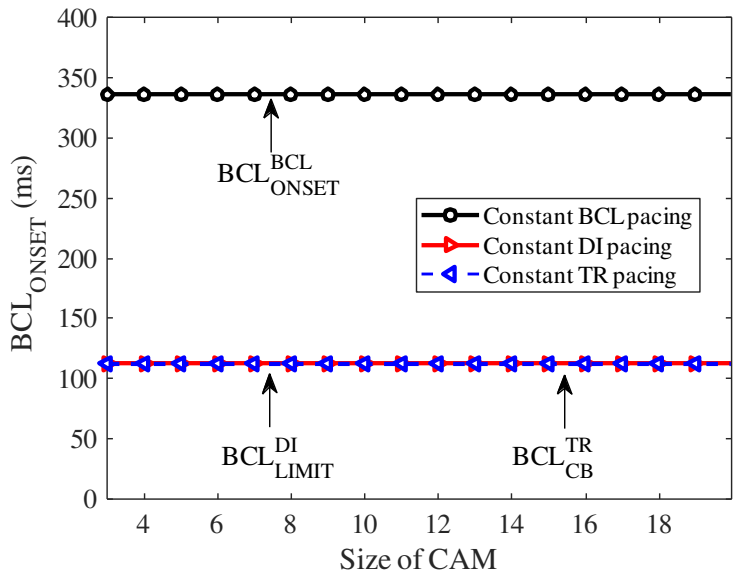

(a)

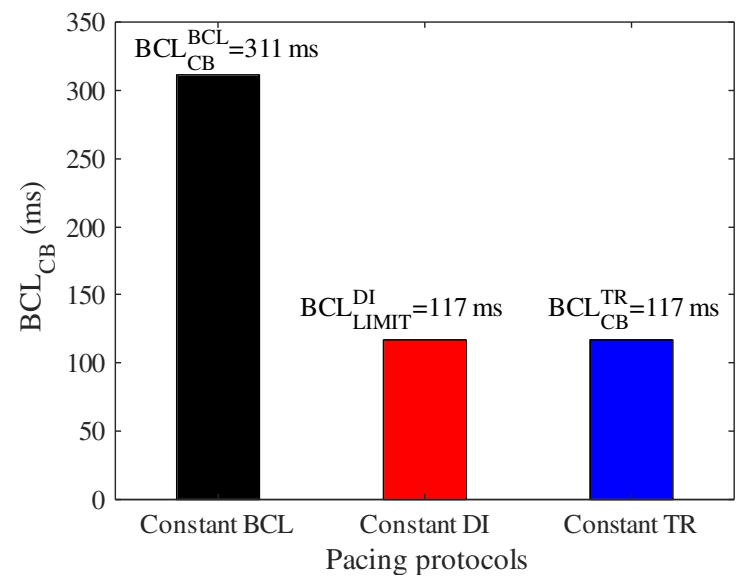

(b)

FIG. 15. Efficacy of different pacing protocols using downsweep protocols with a decrease step $\Delta=1 \mathrm{~ms}$. (a)

Results of $\mathrm{BCL}_{\text {ONSET }}$ under the three pacing protocols as a function of model size. The constant DI pacing protocol is fully effective till the corresponding equivalent $\mathrm{BCL}$ is smaller than $\mathrm{BCL}_{\mathrm{LIMIT}}^{\mathrm{DI}}$. The constant TR pacing protocol can elimite alternans till conduction block occurs at $\mathrm{BCL}_{\mathrm{CB}}^{\mathrm{TR}}$. (b) Results of $\mathrm{BCL}_{\mathrm{CB}}$ under the three pacing protocols.

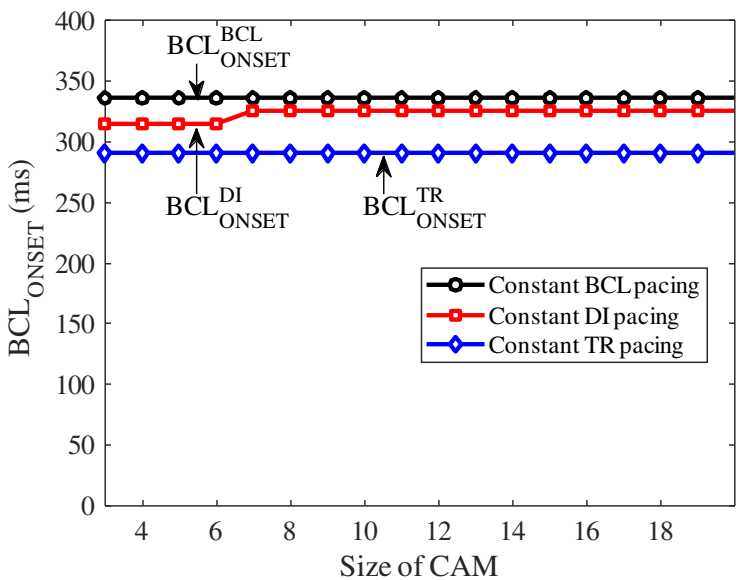

(a)

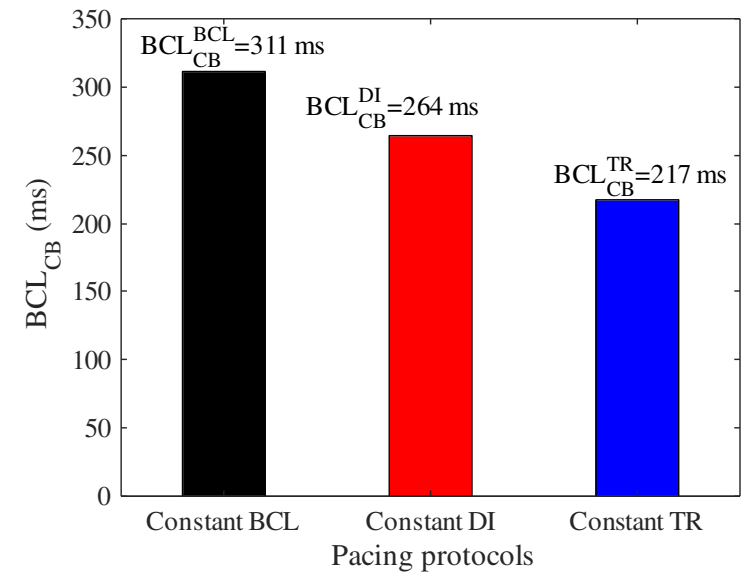

(b)

FIG. 16. Efficacy of different pacing protocols using downsweep protocols with a decrease step $\Delta=5 \mathrm{~ms}$. (a) Results of $\mathrm{BCL}_{\text {ONSET }}$ under the three pacing protocols as a function of model size. (b) Results of $\mathrm{BCL}_{\mathrm{CB}}$ under the three pacing protocols. 


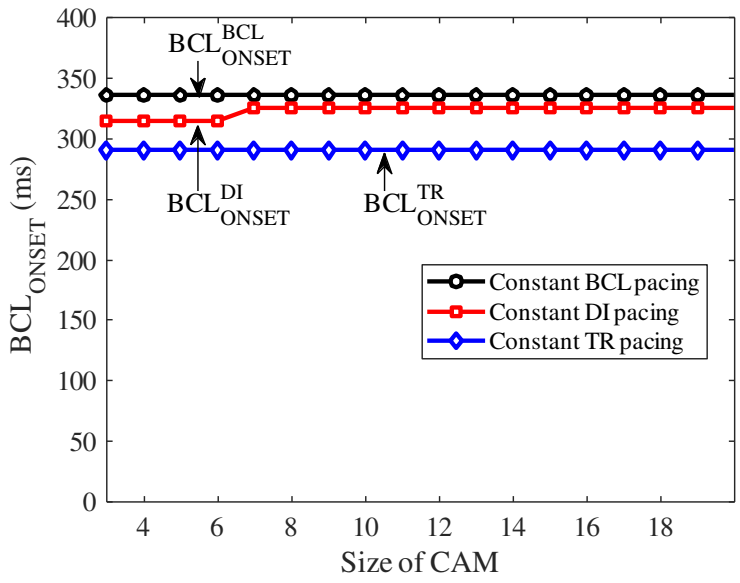

(a)

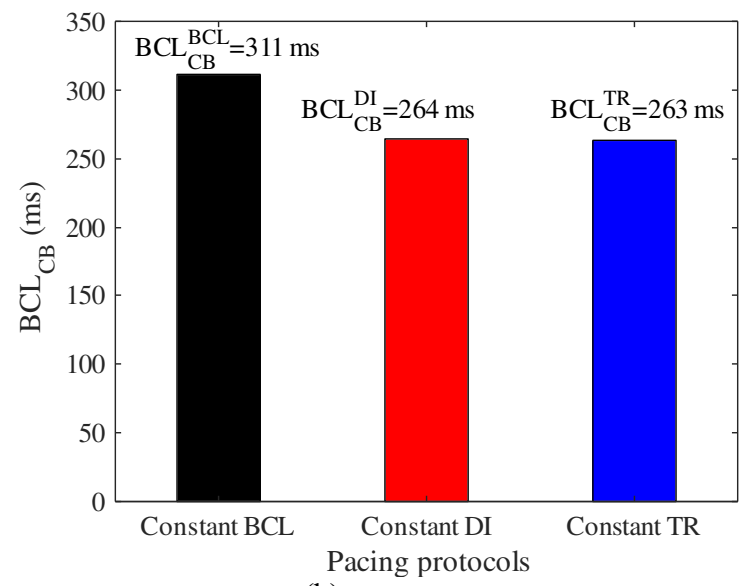

(b)

FIG. 17. Efficacy of different pacing protocols using downsweep protocols with a decrease step $\Delta=10 \mathrm{~ms}$. (a) Results of $\mathrm{BCL}_{\mathrm{ONSET}}$ under the three pacing protocols as a function of model size. (b) Results of $\mathrm{BCL}_{\mathrm{CB}}$ under the three pacing protocols.

Table I: States of cells

\begin{tabular}{ccc}
\hline State & Action potential value & Characteristics \\
\hline Excitation & {$[0.9,1.0] \mathrm{V}$} & Able to excite its neighbors, cannot be stimulated \\
\hline Absolute refractory & {$[0.1,0.9) \mathrm{V}$} & Unable to excite its neighbors, cannot be stimulated \\
\hline Relative refractory & $(0,0.1) \mathrm{V}$ & Unable to excite its neighbors, can be stimulated \\
\hline Resting & $0 \mathrm{~V}$ & \\
\hline
\end{tabular}

